\title{
Report of the Telescope Array - Pierre Auger Observatory Working Group on Energy Spectrum
}

\author{
Dmitri Ivanov $^{a *}$, for the Pierre Auger Collaboration ${ }^{b}$ and the Telescope Array \\ Collaboration ${ }^{c}$ \\ ${ }^{a}$ University of Utah, Department of Physics \& Astronomy and High Energy Astrophysics \\ Institute, Salt Lake City, Utah, USA \\ E-mail: dmiivanov@gmail.com \\ ${ }^{b}$ Observatorio Pierre Auger, Av. San Martín Norte 304, 5613 Malargüe, Argentina \\ E-mail: auger_spokespersons@ fnal.gov \\ Full author list: http://www.auger.org/archive/authors_icrc_2017.html \\ ${ }^{c}$ Telescope Array Project, 201 James Fletcher Bldg, 115 S. 1400 East, Salt Lake City, UT \\ 84112-0830, USA \\ E-mail: ta-icrcecosmic.utah.edu \\ Full author list: http://www.telescopearray.org/index.php/research/collaborators
}

\begin{abstract}
The Telescope Array and Pierre Auger Collaborations have formed a working group to examine the similarities and differences in their measurements of the spectrum of ultra-high energy cosmic rays. The method chosen is for each experiment to measure the spectrum in the declination band of $-15.7^{\circ}<\delta<24.8^{\circ}$, where both experiments have sensitivity. A second step chosen is to correct for the weighting in declination over each experiment's range of sensitivity in the common band of declinations.
\end{abstract}

35th International Cosmic Ray Conference - ICRC2017

10-20 July, 2017

Bexco, Busan, Korea

${ }^{*}$ Speaker. 


\section{Introduction}

The energy spectrum of ultra-high energy cosmic rays (UHECRs) is important for understanding their origin and mechanisms of acceleration and propagation. The UHECR energy spectrum working group (WG) was first proposed at the UHECR-2010 conference in Nagoya, Japan, and a detailed comparison of Pierre Auger Observatory, Telescope Array, Yakutsk, HiRes, and AGASA spectra was presented at the UHECR-2012 conference at CERN [1]. It was concluded that the features seen by all five experiments were consistent among each other after taking into account the differences of their energy scales. For example, at that time, a relative shift of $20 \%$ in energies between the TA and Auger would bring the two results into agreement. It was then understood that the future studies of UHECRs have to rely heavily on the measurements of the Auger Observatory and the Telescope Array, the two largest cosmic ray detectors which use a hybrid design that allows calibrating the energy scale of the surface detector (SD) to the fluorescence detector (FD) on an event by event basis. A fluorescence detector gives a nearly calorimetric estimate of the UHECR energy.

Subsequent results of the WG were reported at the UHECR 2014 conference in Springdale, Utah (USA) [2]. The discussion was focused on the TA and Auger. It was then established that the use of different fluorescence yield models and invisible energy corrections by the two experiments were the largest contributions to the relative energy scale shift, which at that time was estimated to be $16 \%$. The conclusion was that the Auger and TA spectra were in a good agreement in the region around the ankle, while a significant difference became apparent at the highest energies, in the region of the suppression.

In this work, we update the recent results of the WG presented at the UHECR conference held in Kyoto (Japan), in Fall 2016 [3], compare the measurements of the UHECR spectrum by Auger and TA, and discuss their differences.

\section{TA and Auger spectrum}

Auger and TA are the largest cosmic ray detectors in the Southern and the Northern hemispheres, respectively. Auger [4] is located in the province of Mendoza (Argentina), near the town of Malargüe. Auger consists of an SD array of 1,660 water-Cherenkov detectors, arranged on a $1.5 \mathrm{~km}$ spaced triangular grid, with $5 \mathrm{FD}$ stations overlooking the array. The Auger SD effectively covers a $3,000 \mathrm{~km}^{2}$ area on the ground with an altitude of approximately 1,400 m above sea level. The TA experiment is located in Millard County, Utah (USA), in the desert near the town of Delta. The TA SD [5] consists of 507 plastic scintillation counters arranged on a square grid of $1.2 \mathrm{~km}$ spacing, and it is overlooked by 3 FDs [6, 7]. TA SD covers $700 \mathrm{~km}^{2}$ on the ground. The altitude of the TA SD is also approximately $1,400 \mathrm{~m}$ above sea level. In both TA and Auger, the UHECR energy spectrum is measured with best statistics by the SDs.

Auger uses two methods to reconstruct SD events: one for events with zenith angles below $60^{\circ}$, called vertical events, and another for events with zenith angles between $60^{\circ}$ and $80^{\circ}$, called inclined events. Minimum energies are adjusted for each reconstruction type separately, so that the analyses are fully efficient. The minimum energies are $3 \times 10^{18} \mathrm{eV}$ and $4 \times 10^{18} \mathrm{eV}$ for vertical and inclined events, respectively. In TA, the energy spectrum measurement that begins at $10^{18.2} \mathrm{eV}$ 
uses events with zenith angles below $45^{\circ}$ (seen in Figure 1), while the measurement that starts at $10^{19} \mathrm{eV}$ uses events up to $55^{\circ}$ in zenith angle (seen in Figures 3, and 5). The Auger and TA SD energy spectra are shown in the left panel of Figure 1, multiplied by energy cubed to emphasize the changes in the power law. Both Auger and TA clearly see the ankle and the suppression. Evidently, there is an overall energy scale difference between the two measurements, as well as (possibly) energy-dependent differences: if fitted to a broken power law shape, the Auger second break point occurs at $10^{19.62 \pm 0.02} \mathrm{eV}$, while the corresponding break in TA is seen at $10^{19.78 \pm 0.06} \mathrm{eV}$, a factor of $1.4 \pm 0.2$ higher.
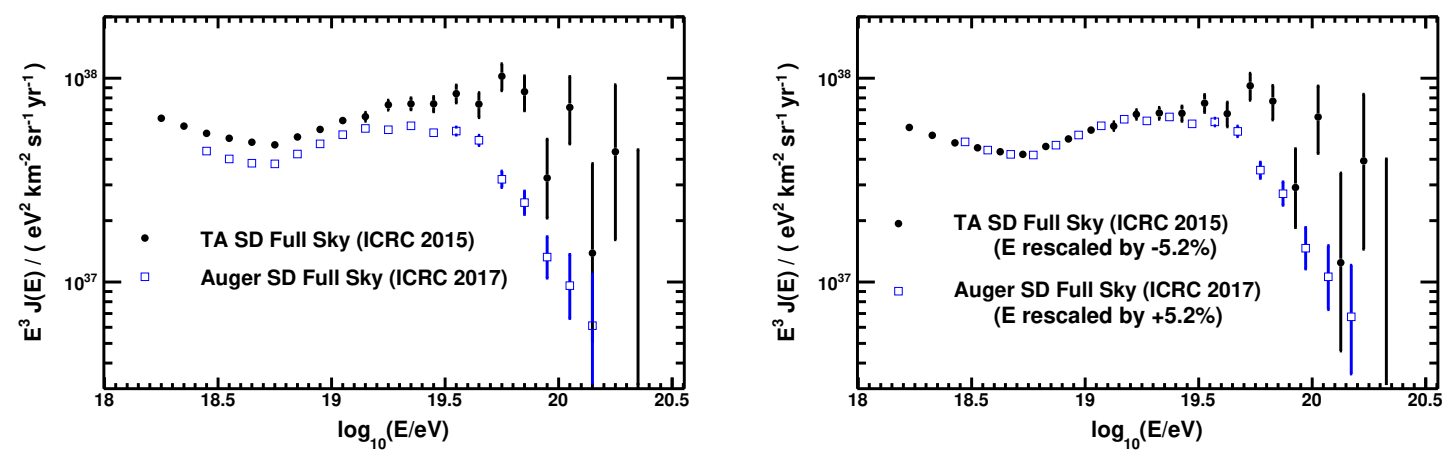

Figure 1: Energy spectrum measurements by the Auger [8] and TA [9] surface detectors. Left: Using energy scales of Auger and TA. Right: TA energy scale is reduced by 5.2\% while Auger energy scale is increased by $5.2 \%$.

As pointed out in Section 1, although the TA and Auger techniques of reconstructing SD event energies are very similar, there do exist differences in their respective instruments and the methods of how the final primary energies are assigned. The systematic uncertainty in the overall energy scale is $14 \%$ for Auger and $21 \%$ for TA, while the uncertainties due to the exposure and the unfolding of the effects of the resolution are subdominant. As the right panel of the Figure 1 shows, the Auger and TA spectra are in a good agreement in the ankle region (from $10^{18.4} \mathrm{eV}$ to $\simeq 10^{19.4} \mathrm{eV}$ ), when the Auger energies are increased by $+5.2 \%$ and the TA energies are reduced by $5.2 \%$. Such shifts are well within the stated uncertainties in the energy scales of both experiments. A large difference remains above $\simeq 10^{19.5} \mathrm{eV}$, in the region of the suppression.

The sources of differences in the energy scales of both experiments, as well as the exposure and resolution unfolding calculations, have been cross-checked in the UHECR-2014 meeting. In the WG report of UHECR-2016, and in this work, we focus on the remaining difference in the region of the high energy suppression. To determine whether this difference is an instrumental or an astrophysical effect, we have performed a comparison of Auger and TA spectra in the common declination band, a range of declination values that is in the field of view of both experiments: $-15.7^{\circ}<\delta<24.8^{\circ}$. In this work, we use the Auger and TA analyses with upper limits on the event zenith angles of $60^{\circ}$ and $55^{\circ}$, respectively. Moreover, for the purposes of this comparison, we use a new spectrum calculation technique that takes into account the details of the Auger and TA exposure dependence on the declination [3]. 


\section{Comparison of the TA and Auger spectra in the common declination band}

Figure 2 shows the directional exposures of the two experiments. For the Auger vertical anal-

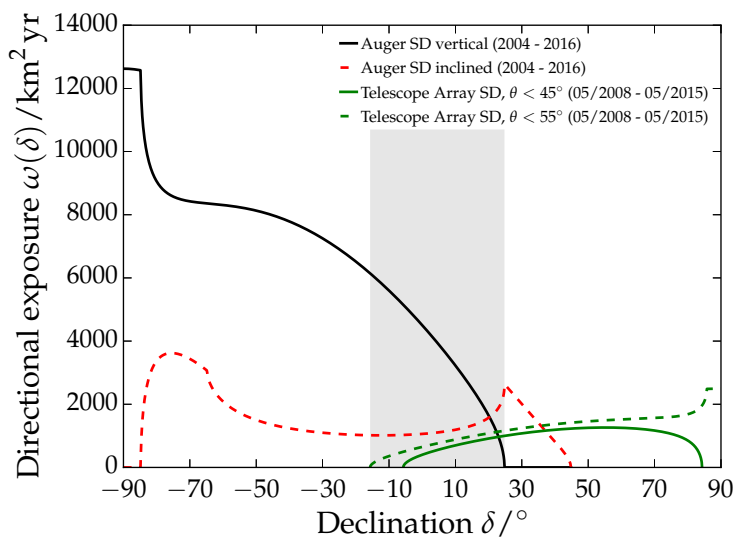

Figure 2: Auger and TA exposure plotted versus declination. For Auger, shown are the exposure values for the vertical (black solid line) and inclined (dashed red line) analyses. For TA, the exposure is shown for the maximum zenith angle cuts of $45^{\circ}$ (green solid line) and $55^{\circ}$ (green dashed line). Gray shaded area shows the TA and Auger common declination band, which extends from $-15.7^{\circ}$ to $24.8^{\circ}$.

ysis that covers declinations from $-90^{\circ}$ to $24.8^{\circ}$, the total exposure is $51,588 \mathrm{~km}^{2}$ sr yr. For TA analysis with event zenith angles extending to $55^{\circ}$, the total exposure is $8,300 \mathrm{~km}^{2} \mathrm{sr}$ yr. TA is sensitive in the declination range from $-16^{\circ}$ to $90^{\circ}$.

In order to calculate and compare the Auger and TA spectra in the common declination band (shaded area in Figure 2), we use the following method:

$$
J_{1 / \omega}(E)=\frac{1}{\Delta \Omega \Delta E} \sum_{i=1}^{N} \frac{1}{\omega\left(\delta_{i}\right)}
$$

where $J_{1 / \omega}(E)$ is the differential flux $J$ as a function of energy $E$, calculated by this " $1 / \omega-$ method", $\Delta \Omega=2 \pi \int_{\delta_{\min }}^{\delta_{\max }} \mathrm{d} \delta \cos (\delta)$ is the solid angle covered by the common declination band $\left(\delta_{\min }=-15.7^{\circ}, \delta_{\max }=24.8^{\circ}\right), \omega$ is the directional exposure shown in Figure 2 , and $\delta_{i}$ is the declination of the $i^{t h}$ event. The sum is over $N$ events in the energy interval $\Delta E$. The statistical uncertainty of $J_{1 / \omega}(E)$, if one were to ignore any anisotropies, is given by (to first order):

$$
\Delta J_{1 / \omega}(E)=\frac{1}{\Delta \Omega \Delta E}\left\{\frac{\int_{\delta_{\min }}^{\delta_{\max }} \mathrm{d} \delta \cos (\delta) / \omega(\delta)}{\int_{\delta_{\min }}^{\delta_{\max }} \mathrm{d} \delta \omega(\delta) \cos (\delta)}\right\}^{1 / 2} \sqrt{N}
$$

For the formal derivation of this method, as well as the cross-checks with the standard TA and Auger spectrum calculation techniques, please see [3]. The Left panel of Figure 3 shows the results with Auger and TA energies scaled by $\pm 5.2 \%$ (as in the right panel of Figure 1). When we fit the two results, $J_{1 / \omega}^{\text {Auger }}$ and $J_{1 / \omega}^{\mathrm{TA}}$, to broken power law functions, the high energy cutoff points become only $0.6 \sigma$ different: $\log _{10}(E / \mathrm{eV})=19.59 \pm 0.04$ for TA, and $\log _{10}(E / \mathrm{eV})=19.56 \pm 0.03$ for 

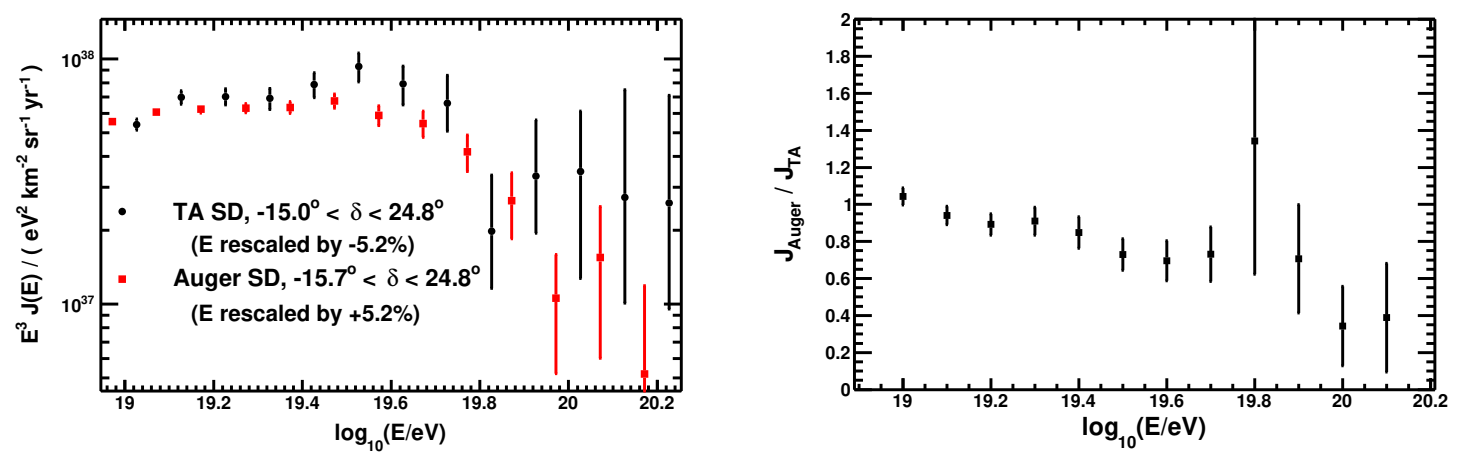

Figure 3: Left: Auger SD (red squares) and the TA SD (black circles) spectra in the common declination band, calculated using the $1 / \omega$ method. The Auger energy scale has been increased by $5.2 \%$ while the TA energies have been decreased by $5.2 \%$, as it was done in the right panel of Figure 1. Right: Ratio of the Auger $\left(J_{\text {Auger }}\right)$ and TA $\left(J_{\mathrm{TA}}\right)$ fluxes that have been calculated using the $1 / \omega$ method.

Auger. This agreement is an important step towards comparing the results of the two experiments. However, differences still remain, as it can be seen in the right panel of the Figure 3: the ratio of fluxes $J_{1 / \omega}^{\text {Auger }} / J_{1 / \omega}^{\mathrm{TA}}$, in the common declination band, depends on energy in a significant way.

We have also examined possible declination dependencies of the spectra within each individual experiment. In the case of Auger, as Figure 4 shows, the spectrum does not appear to depend on the declination in a significant way. All three Auger spectra, for declinations $-90^{\circ}<\delta<$

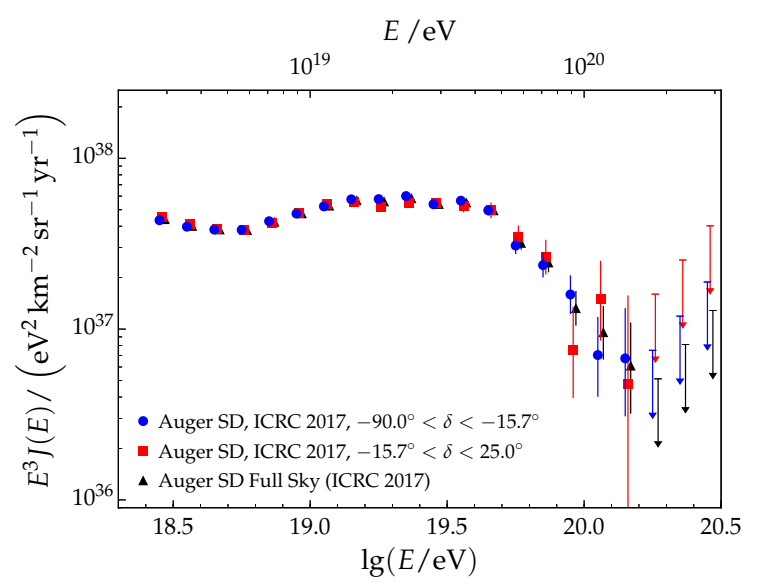

Figure 4: Auger spectra covering the sky to the south of the common declination band (shown in blue), common declination band (shown in red), and the combination of the two (shown in black).

$-15.7^{\circ},-15.7^{\circ}<\delta<25.0^{\circ}$, and $-90.0^{\circ}<\delta<25.0^{\circ}$, are in a good agreement among each other. In the case of TA, on the other hand, the situation is different: as Figure 5 shows, the break point (using the broken power law fit) in the spectrum for lower declinations $-16^{\circ}<\delta<24.8^{\circ}$ occurs at $\log _{10}(E / \mathrm{eV})=19.59 \pm 0.06$, while for $24.8^{\circ}<\delta<90^{\circ}$, the second break point occurs at $\log _{10}(E / \mathrm{eV})=19.85 \pm 0.03$. It should be noted that although the spectrum calculations in 


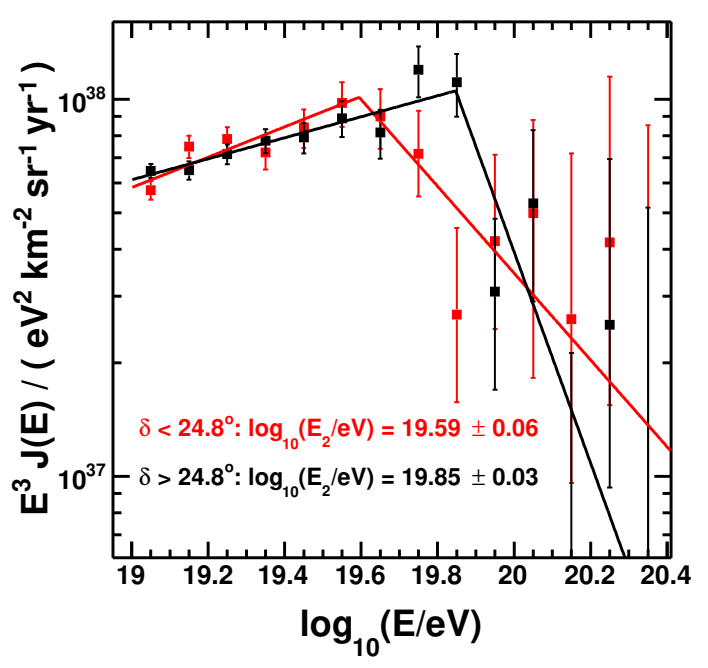

Figure 5: TA surface detector spectra for two declination bands: common declination band (in red) and over the rest of the northern hemisphere (in black).

Figures 4, 5 used traditional Auger and TA methods, cross-checks have been made [8, 9], and it was shown that the $1 / \omega$ method produced similar results [3].

\section{Summary}

We have reviewed and compared the results of the UHECR spectra measured by the Pierre Auger and Telescope Array experiments. It was established that scaling the energies of Auger and TA by $+5.2 \%$ and $-5.2 \%$, respectively, brings the two measurements into a good agreement around the ankle region from $10^{18.4} \mathrm{eV}$ to $\simeq 10^{19.4} \mathrm{eV}$. Energy scaling of 5.2\% is well within the systematic uncertainties stated by the experiments. At the energies around the suppression region, on the other hand, we have found that even after restricting the comparison to the region of the sky that is observed by both experiments, despite a noticeable improvement in the agreement of the spectra, statistically significant differences were still present. We have not identified the sources of the remaining discrepancies at this time. 


\section{References}

[1] B.R. Dawson et al., for the Telescope Array and Pierre Auger Collaborations, EPJ Web of Conferences 53 (2013) 01005.

[2] I. Mariş et al., for the Telescope Array and Pierre Auger Collaborations, talk given at the 2014 Conference on Ultrahigh Energy Cosmic Rays, Springdale, Utah (USA).

[3] T. AbuZayyad et al., for the Telescope Array and Pierre Auger Collaborations, proceedings of the 2016 Conference on Ultrahigh Energy Cosmic Rays, Kyoto (Japan).

[4] The Pierre Auger Collaboration, Nucl. Instrum. Meth. A 798 (2015) 172.

[5] T. Abu-Zayyad et al., Nucl. Instrum. Meth. A 689 (2013) 87.

[6] T. Abu-Zayyad et al., Nucl. Instrum. Meth. A 676 (2012) 54.

[7] T. Abu-Zayyad et al., Astropart. Phys. 109 (2012) 39.

[8] F. Fenu, for the Pierre Auger Collaboration, these proceedings.

[9] D. Ivanov, for the Telescope Array Collaboration, these proceedings. 